\title{
Association between the XRCC1 Polymorphisms and Thyroid Cancer Risk: A Meta-Analysis from Case-Control Studies
}

\author{
Fei-Fei $\mathbf{W u}^{1}$, Xiao-Feng $\mathrm{He}^{2}$, Hu-Wei Shen ${ }^{3}$, Gui-Jun Qin ${ }^{1 *}$ \\ 1 Department of Endocrinology, First Affiliated Hospital of Zhengzhou University, Zhengzhou, China, 2 Department of Research, Peace Hospital of Changzhi Medical \\ College, Changzhi, China, 3 Department of Endocrinology, Peace Hospital of Changzhi Medical College, Changzhi, China
}

\begin{abstract}
Background: The previous published data on the association between the X-ray repair cross-conplementation group 1 (XRCC1) polymorphisms and thyroid cancer risk remained controversial. Hence, we performed a meta-analysis on all available studies that provided 1729 cases and 3774 controls (from 11 studies) for XRCC1 Arg399Gln, 1040 cases and 2487 controls for Arg194Trp (from 7 studies), and 1432 cases and 3356 controls for Arg280His (from 8 studies).

Methodology/Principal Findings: PubMed, CNKI, and EMBASE database were searched to identify relevant studies. Overall, no significant association was found between XRCC1 Arg399Gln (recessive model: $\mathrm{OR}=0.95,95 \% \mathrm{Cl}=0.77-1.15$; dominant model: $\mathrm{OR}=0.89,95 \% \mathrm{Cl}=0.75-1.05$; homozygote model: $\mathrm{OR}=0.92,95 \% \mathrm{Cl}=0.69-1.23$; Heterozygote model: OR $=0.91$, $95 \% \mathrm{Cl}=0.80-1.03$; additive model: $\mathrm{OR}=0.93,95 \% \mathrm{Cl}=0.81-1.07$ ), Arg194Trp (recessive model: $\mathrm{OR}=1.41,95 \% \mathrm{Cl}=0.62-$ 3.23; dominant model: $\mathrm{OR}=1.01,95 \% \mathrm{Cl}=0.77-1.34$; homozygote model: $\mathrm{OR}=1.42,95 \% \mathrm{Cl}=0.55-3.67$; Heterozygote model: $\mathrm{OR}=1.03,95 \% \mathrm{Cl}=0.85-1.26$; additive model: $\mathrm{OR}=1.08,95 \% \mathrm{Cl}=0.81-1.42$ ), and Arg280His (recessive model: $\mathrm{OR}=1.08,95 \% \mathrm{Cl}=0.56-2.10$; dominant model: $\mathrm{OR}=1.01,95 \% \mathrm{Cl}=0.84-1.22$; homozygote model: $\mathrm{OR}=1.00,95 \% \mathrm{Cl}=0.51-$ 1.96; Heterozygote model: $\mathrm{OR}=1.04,95 \% \mathrm{Cl}=0.75-1.42$; additive model: $\mathrm{OR}=1.03,95 \% \mathrm{Cl}=0.86-1.23$ ) and thyroid cancer risk when all the eligible studies were pooled into the meta-analysis. In the further stratified and sensitivity analyses, significant association was still not found in these three genetic polymorphisms.
\end{abstract}

Conclusions/Significance: In summary, this meta-analysis indicates that XRCC1 Arg399GIn, Arg280His, and Arg194Trp are not associated with thyroid cancer.

Citation: Wu F-F, He X-F, Shen H-W, Qin G-J (2014) Association between the XRCC1 Polymorphisms and Thyroid Cancer Risk: A Meta-Analysis from Case-Control Studies. PLoS ONE 9(9): e87764. doi:10.1371/journal.pone.0087764

Editor: Jacques Emile Dumont, Universite Libre de Bruxelles (ULB), Belgium

Received September 26, 2013; Accepted December 1, 2013; Published September 11, 2014

Copyright: (c) 2014 Wu et al. This is an open-access article distributed under the terms of the Creative Commons Attribution License, which permits unrestricted use, distribution, and reproduction in any medium, provided the original author and source are credited.

Funding: The authors have no funding or support to report.

Competing Interests: The authors have declared that no competing interests exist.

* Email: qinguijun123@163.com

\section{Introduction}

Thyroid carcinomas are the most frequent endocrine malignancies which among these thyroid carcinomas, more than 90 percent are differentiated thyroid carcinomas (DTC). Pathologically, DTG include papillary, follicular, and Hürthle cell carcinoma [1]. To date, exposure to ionizing radiation is the only known risk factor for thyroid cancer [2]. However, there are evidences that some gene variants including DNA repair genes influence on DTG susceptibility. XRCG1 is one of the candidate genes which its variant relationship with thyroid cancer has not been extensively studied [3].

The XRCC (X-Ray cross-complementing) genes were initially discovered through their role in DNA damage response caused by ionizing radiation. They are important components of various DNA repair pathways contributing to DNA-damage processing and genetic stability [4]. X-ray cross-complementing gene 1 (XRCC1) is involved in the repair of DNA base damage and singlestrand DNA breaks by binding DNA ligase III at its carboxyl and DNA polymerase $\beta$ and poly (ADP-ribose) polymerase at the site of damaged DNA [5] and is known to participate in base excision repair (BER) of small lesions such as oxidized or reduced bases, fragmented or nonbulky adducts, and lesions caused by methylating agents [6]. Three common polymorphisms within the XRCC1 have been identified at codon 194, 280, and 399 (Arg194Trp, Arg280His, and Arg399Gln) [7].

Many studies have reported the association of XRCC1 polymorphisms at 194, 280, and 399 (Arg194Trp, Arg280His, and Arg399Gln) with thyroid cancer risk [16-25], but the results were inconclusive, some original studies thought that these polymorphisms were associated with thyroid cancer risk, but others had different opinions. In addition, attention has been mainly drawn at a meta-analytical level upon the association of XRCC1 polymorphisms at 194, 280, and 399 with thyroid cancer risk [8,9]. However, the previous meta-analyses on XRCC1 Arg194Trp, Arg280His, and Arg399Gln with thyroid cancer risk have shown conflicting conclusions. In order to explore the association between Arg399Gln, Arg194Trp, and Arg280His polymorphisms with thyroid cancer risk, an updated meta-analysis was conducted to summarize the data. Meta-analysis is a good 
method for summarizing the different studies. It can not only overcome the problem of small size and inadequate statistical power of genetic studies of complex traits, but also provide more reliable results than a single case-control study.

\section{Materials and Methods}

\section{Identification and eligibility of relevant studies}

A bibliographical search was performed in PubMed, CNKI, and EMBASE database to identify studies that evaluated XRCG1 polymorphisms and thyroid cancer up to April 10, 2014. The search terms used were: (polymorphism or mutation or variant) and (XRCG1 or "X-ray repair cross-conplementation group 1") and thyroid. The search was not limited to language. Additional studies were identified by hand searching references in original articles and review articles. Authors were contacted directly regarding crucial data not reported in original articles. In addition, studies were identified by a manual search of the reference lists of reviews and retrieved studies. We included all the case- ${ }^{-}$control studies and cohort studies that investigated the association between XRCC1 Arg399Gln, Arg194Trp, and Arg280His polymorphisms and thyroid cancer risk with genotyping data. All eligible studies were retrieved, and their bibliographies were checked for other relevant publications. When the same sample was used in several publications, only the most complete information was included following careful examination.

\section{Inclusion criteria}

The included studies needed to have met the following criteria: (1) only the case-control studies or cohort studies were considered, (2) evaluated the XRCC1 Arg399Gln, Arg194Trp, and Arg280His polymorphisms and thyroid cancer risk, and (3) the genotype distribution of the polymorphisms in cases and controls were described in details and the results were expressed as odds ratio (OR) and corresponding 95\% confidence interval (95\% CI). Major reasons for exclusion of studies were as follows: (1) not for cancer research, (2) only case population, and (3) duplicate of previous publication.

\section{Data extraction}

Information was carefully extracted from all eligible studies independently by two investigators according to the inclusion criteria listed above. The following data were collected from each study: first author's name, year of publication, country of origin, ethnicity, source of controls, genotyping method, and numbers of cases and controls in the XRGC1 Arg399Gln, Arg194Trp, and Arg280His genotypes whenever possible. Ethnicity was categorized as "Caucasian," "African," (including African Americans) and "Asian." We considered the samples of studies from India and Pakistan as of "Indian"" ethnicity, and samples from Middle Eastern countries as "Middle Eastern" ethnicity. When one study did not state which ethnic groups was included or if it was impossible to separate participants according to phenotype, the sample was termed as "mixed population." We did not define any minimum number of patients to include in this meta-analysis. Articles that reported different ethnic groups and different countries or locations, we considered them different study samples for each category cited above.

\section{Statistical analysis}

Crude odds ratios (ORs) together with their corresponding 95\% CIs were used to assess the strength of association between the XRCC1 Arg399Gln, Arg194Trp, and Arg280His polymorphisms and thyroid cancer risk. The pooled ORs were performed for dominant model (Arg399Gln: Arg/Gln+Gln/Gln versus Arg/Arg, Arg194Trp: Arg/Trp+Trp/Trp versus Arg/Arg, and Arg280His: Arg/Gln+His/His versus Arg/Arg); recessive model (Arg399Gln: Gln/Gln versus Arg/Gln+Arg/Arg, Arg 194Trp: Trp/Trp versus Arg/Trp+Arg/Arg, and Arg280His: His/His versus Arg/His+ Arg/Arg); Homozygote model (Arg399Gln: Gln/Gln versus Arg/ Arg, Arg 194Trp: Trp/Trp versus Arg/Arg, and Arg280His: His/ His versus Arg/Arg), Heterozygote model (Arg399Gln: Arg/Gln versus Arg/Arg, Arg194Trp: Arg/Trp versus Arg/Arg, and Arg280His: Arg/Gln versus Arg/Arg), and additive model (Arg399Gln: Gln versus Arg, Arg194Trp: Trp versus Arg, and Arg280His: His versus Arg), respectively. Heterogeneity assumption was checked by a chi-square-based $Q$ test (Heterogeneity was considered statistically significant if $P<0.10$ ) [26] and quantified using the $I^{2}$ value, a value that describes the percentage of variation across studies that are due to heterogeneity rather than chance, where $I^{2}=0 \%$ indicates no observed heterogeneity, with $25 \%$ regarded as low, $50 \%$ as moderate, and $75 \%$ as high [27]. If results were not heterogeneous, the pooled ORs were calculated by the fixed-effect model (we used the $Q$-statistic, which represents the magnitude of heterogeneity between-studies) [28]. Otherwise, a random-effect model was used (when the heterogeneity betweenstudies were significant) [29]. In addition to the comparison among all subjects, we also performed stratification analyses by ethnicity and histological subtype (papillary thyroid cancer and follicular thyroid cancer). Moreover, the extent to which the combined risk estimate might be affected by individual studies was assessed by consecutively omitting every study from the metaanalysis (leave-one-out sensitivity analysis). This approach would also capture the effect of the oldest or first positive study (first study effect). In addition, sensitivity analysis was also performed, excluding studies whose allele frequencies in controls exhibited significant deviation from the Hardy-Weinberg equilibrium (HWE), given that the deviation may denote bias. Deviation of HWE may reflect methodological problems such as genotyping errors, population stratification or selection bias. HWE was calculated by using the goodness-of-fit test, and deviation was considered when $P<0.05$. Begg's funnel plots [30] and Egger's linear regression test [31] were used to assess publication bias. A meta-regression analysis was carried out to identify the major sources of between-studies variation in the results, using the log of the ORs from each study as dependent variables, and ethnicity and source of controls as the possible sources of heterogeneity. All of the calculations were performed using STATA version 10.0 (STATA Corporation, College Station, TX).

\section{Results}

\section{Literature Search and Meta-analysis Databases}

Relevant publications were retrieved and preliminarily screened. As shown in Fig. 1, 45 publications were identified, among which 17 irrelevant papers were excluded. Thus, 28 publications were eligible. Among these publications, 17 articles were excluded because they were review articles, case reports, and other polymorphisms of XRCC1. In addition, one was excluded because the data of genotyping distribution was missing [32]. As summarized in Table 1, 10 articles with 25 case-control studies publications were selected in the final meta-analysis, including 1729 cases and 3774 controls for XRCC1 Arg399Gln (from 11 studies), 1,040 cases and 2,487 controls for Arg194Trp (from 7 studies), 1,432 cases and 3,356 controls for Arg280His (from 8 studies). Tables 1 list all essential information such as the publication year, first author, Country, ethnicity, source of controls, and Genotyping method for XRGCl Arg399Gln, 
Arg 194Trp, and Arg280His, respectively. Genotype frequencies for thyroid cancer cases and controls were listed in Table 2-4. Among these, two separated case-control studies were included from Akulevich et al. [19] and were considered separately. And one publication was analyzed only in dominant model because Sigurdson et al. [25] provide the limited genotyping information for two XRCCl polymorphisms (Arg194Trp and Arg280His). Among them, six studies focused on PTC $(18,20,22,24,25)$ and only Santos et al. [16] on both PTC and FTC. All of the cases were pathologically confirmed.

\section{Quantitative synthesis}

Table 5 listed the main results of the meta-analysis of XRCC1 polymorphisms and thyroid cancer risk. For Arg399Gln, there was no significant association between this polymorphism and thyroid cancer risk in any genetic model when all the eligible studies were pooled together. Similarly, the combined results did not showed any association between Arg194Trp/Arg280His polymorphisms and thyroid cancer risk for all genetic models. However, in the subgroup analysis by ethnicity, the results showed that Arg/His genotype was associated with an increased risk of thyroid cancer among Caucasians (dominant model: $\mathrm{OR}=1.43,95 \% \mathrm{CI}=1.08-$ $1.89, P$ value of heterogeneity test $\left[P_{\mathrm{h}}\right]=0.513, I^{2}=0.0 \%$; additive model: $\mathrm{OR}=1.38,95 \% \mathrm{CI}=1.05-1.80, P_{\mathrm{h}}=0.551$, $I^{2}=0.0 \%$; Heterozygote model: $\mathrm{OR}=1.45,95 \% \mathrm{CI}=1.09$ $\left.1.93, P_{\mathrm{h}}=0.495, I^{2}=0.0 \%\right)$. And carriers of the $399 \mathrm{Gln}$ variant allele have a decreased thyroid cancer risk in mixed population (dominant model: $\mathrm{OR}=0.73,95 \% \mathrm{CI}=0.55-0.97, P_{\mathrm{h}}=0.326$, $I^{2}=0.0 \%$; additive model: $\mathrm{OR}=0.73,95 \%$ CI $=0.59-0.92$, $P_{\mathrm{h}}=0.308, \quad I^{2}=3.6 \%$; recessive model: $\mathrm{OR}=0.56, \quad 95 \%$ $\mathrm{CI}=0.34-0.93, \quad P_{\mathrm{h}}=0.588, \quad I^{2}=0.0 \% ;$ homozygote model: $\left.\mathrm{OR}=0.50,95 \% \mathrm{CI}=0.30-0.85, P_{\mathrm{h}}=0.460, I^{2}=0.0 \%\right)$. We also detected that the Trp allele of Arg194Trp polymorphism was significantly increased thyroid cancer risk in mixed population (additive model: $\mathrm{OR}=1.49,95 \% \mathrm{CI}=1.02-2.17$ ). When subgroup analysis by histological subtype, the results showed that Arg 194Trp polymorphism was associated with decreased papillary

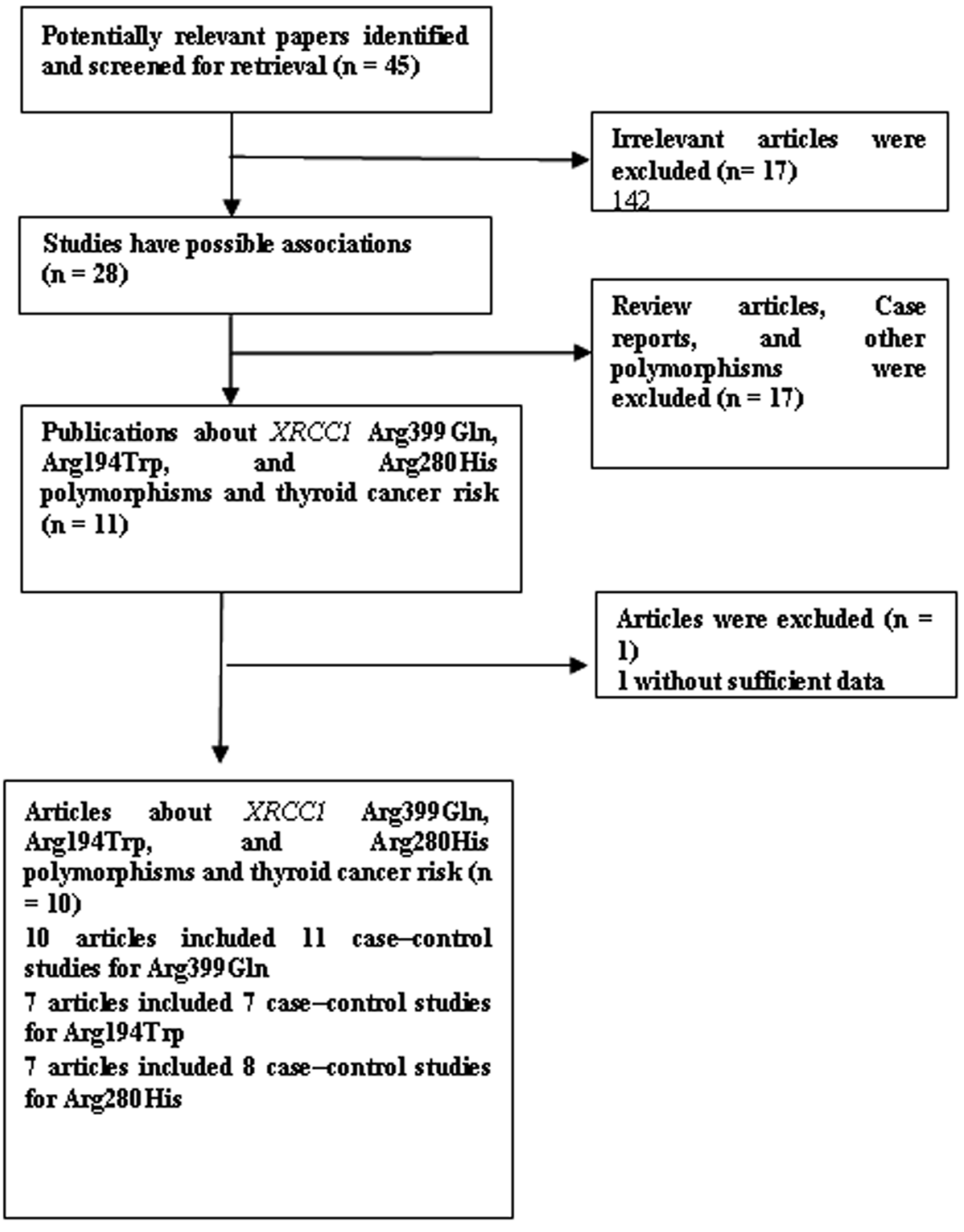

Figure 1. Study flow chart explaining the selection of the $\mathbf{1 0}$ eligible articles included in the meta-analysis. doi:10.1371/journal.pone.0087764.g001 
Table 1. Characteristics of studies included in the meta-analysis.

\begin{tabular}{|c|c|c|c|c|c|c|}
\hline First author & Year & Country & Ethnicity & SC & SNP & Genotyping method \\
\hline Santos [16] & 2012 & Portugal & Caucasian & $\mathrm{HB}$ & Arg399Gln & PCR-RFLP \\
\hline Santos [16] & 2012 & Portugal & Caucasian & $\mathrm{HB}$ & Arg194Trp & PCR-RFLP \\
\hline Fard-Esfahani [17] & 2011 & Iran & Caucasian & $\mathrm{HB}$ & Arg399Gln & PCR-RFLP \\
\hline Fard-Esfahani [17] & 2011 & Iran & Caucasian & $\mathrm{HB}$ & Arg194Trp & PCR-RFLP \\
\hline Fard-Esfahani [17] & 2011 & Iran & Caucasian & $\mathrm{HB}$ & Arg280His & PCR-RFLP \\
\hline Ryu [18] & 2011 & Korea & Asian & $\mathrm{HB}$ & Arg399GIn & PCR-RFLP \\
\hline Ryu [18] & 2011 & Korea & Asian & $\mathrm{HB}$ & Arg194Trp & PCR-RFLP \\
\hline García-Quispes [19] & 2011 & Spain & Caucasian & $\mathrm{HB}$ & Arg399Gln & iPLEX \\
\hline García-Quispes [19] & 2011 & Spain & Caucasian & $\mathrm{HB}$ & $\mathrm{Arg} 280 \mathrm{His}$ & iPLEX \\
\hline Akulevich [20] & 2009 & $\mathrm{RB}$ & Caucasian & $\mathrm{HB}$ & Arg399Gln & PCR-RFLP \\
\hline Akulevich [20] & 2009 & $\mathrm{RB}$ & Caucasian & PB & Arg399GIn & PCR-RFLP \\
\hline Akulevich [20] & 2009 & RB & Caucasian & $\mathrm{HB}$ & Arg280His & PCR-RFLP \\
\hline Akulevich [20] & 2009 & $\mathrm{RB}$ & Caucasian & PB & Arg280His & PCR-RFLP \\
\hline Ho [21] & 2009 & USA & Mixed & $\mathrm{HB}$ & Arg399Gln & PCR-RFLP \\
\hline Ho [21] & 2009 & USA & Mixed & $\mathrm{HB}$ & Arg194Trp & PCR-RFLP \\
\hline Ho [21] & 2009 & USA & Mixed & $\mathrm{HB}$ & Arg280His & PCR-RFLP \\
\hline Siraj [22] & 2009 & Saudi & ME & $\mathrm{HB}$ & Arg399Gln & PCR-RFLP \\
\hline Siraj [22] & 2009 & Saudi & ME & $\mathrm{HB}$ & Arg280His & PCR-RFLP \\
\hline Chiang [23] & 2008 & China & Asian & $\mathrm{HB}$ & Arg399Gln & Taqman \\
\hline Chiang [23] & 2008 & China & Asian & $\mathrm{HB}$ & Arg194Trp & Taqman \\
\hline Chiang [23] & 2008 & China & Asian & $\mathrm{HB}$ & Arg280His & Taqman \\
\hline Zhu [24] & 2004 & China & Asian & $\mathrm{HB}$ & Arg399Gln & PCR-RFLP \\
\hline Zhu [24] & 2004 & China & Asian & $\mathrm{HB}$ & Arg194Trp & PCR-RFLP \\
\hline Sigurdson [25] & 2009 & Kazakhstan & Mixed & $\mathrm{N}$ & Arg399Gln & Taqman \\
\hline Sigurdson [25] & 2009 & Kazakhstan & Mixed & $\mathrm{N}$ & Arg194Trp & Taqman \\
\hline Sigurdson [25] & 2009 & Kazakhstan & Mixed & $\mathrm{N}$ & Arg280His & Taqman \\
\hline
\end{tabular}

HT, Histological type; RB Russia and Belarus, HB hospital-based studies, N nested case-control studies, PB population-based studies, SC source of controls, doi:10.1371/journal.pone.0087764.t001

thyroid cancer $(\mathrm{PTC})$ risk in dominant model $(\mathrm{OR}=0.71,95 \%$ $\mathrm{CI}=0.50-0.99, P_{\mathrm{h}}=0.525, I^{2}=0.0 \%$ ).

\section{Test of heterogeneity and sensitivity}

There was significant heterogeneity among these studies for dominant model comparison (Arg399Gln: $P_{\mathrm{h}}=0.089$, Arg 194Trp: $P_{\mathrm{h}}=0.088$, and Arg280His: $\left.P_{\mathrm{h}}=0.061\right)$, recessive model (Arg194Trp: $P_{\mathrm{h}}=0.041$ ), homozygote model comparison (Arg399Gln: $P_{\mathrm{h}}=0.090, \operatorname{Arg} 194 \mathrm{Trp}: P_{\mathrm{h}}=0.014$ ), heterozygote model (Arg280His: $P_{\mathrm{h}}=0.035$ ), and additive model comparison (Arg399Gln: $P_{\mathrm{h}}=0.031$, Arg194Trp: $P_{\mathrm{h}}=0.019$ ). Then, we assessed the source of heterogeneity by ethnicity and source of controls. The results of meta-regression indicated that ethnicity (dominant model: $P=0.039$ for Arg399Gln and $P=0.001$ for Arg280His; additive model: $P=0.001$ for Arg399Gln; homozygote model: $P=0.002$ for Arg399Gln; heterozygote model: $P<$ 0.001 for Arg280His) but not source of controls (dominant model: $P=0.799$ for Arg399Gln and $P=0.086$ for Arg280His; additive model: $P=0.500$ for Arg399Gln; homozygote model: $P=0.388$ for Arg399Gln; heterozygote model: $P=0.159$ for Arg280His) contributed to substantial heterogeneity among the meta-analysis. Although there were two studies [18,25] deviated from HWE for Arg399Gln polymorphism, the corresponding pooled ORs were not materially altered by excluding these studies in overall and subgroup analyses. However, when the study of Ho et al. [21] was excluded, the results were changed in mixed population for Arg399Gln (dominant model: $\mathrm{OR}=1.06,95 \%$ CI $=0.47-2.40$; additive model: $\mathrm{OR}=1.00,95 \% \mathrm{CI}=0.53-1.88$; recessive model: $\mathrm{OR}=0.82,95 \% \mathrm{CI}=0.19-3.55$; homozygote model: $\mathrm{OR}=0.86$, 95\% CI $=0.19-3.92)$.

For Arg194Trp polymorphism, when one study was excluded, the results were also changed in mixed population (data not shown) and PTC (dominant model: OR $=0.85,95 \%$ CI $=0.55-$ 1.29). For Arg280His polymorphism, when one study was excluded, the results were also changed in Caucasians (dominant model: $\quad \mathrm{OR}=1.25, \quad 95 \% \quad \mathrm{CI}=0.84-1.85 ;$ additive model: $\mathrm{OR}=1.21, \quad 95 \% \quad \mathrm{CI}=0.83-1.76 ; \quad$ Heterozygote model: $\mathrm{OR}=1.28,95 \% \mathrm{CI}=0.86-1.90)$.

\section{Publication bias}

Both Begg's funnel plot and Egger's test were performed to access the publication bias of this meta-analysis. Begg's funnel plots did not reveal any evidence of obvious asymmetry in any genetic model in the overall meta-analysis (Figure 2-4). The Egger's test results also suggested no evidence of publication bias in the meta-analysis of $\operatorname{Arg} 399 \mathrm{Gln}(P=0.523$ for dominant model, $P=0.466$ for recessive model, $P=0.796$ for additive model, $P=0.598$ for homozygote model, and $P=0.329$ for heterozygote model), Arg 194Trp $(P=0.224$ for dominant model, $P=0.758$ for recessive model, $P=0.618$ for additive model, $P=0.822$ for 


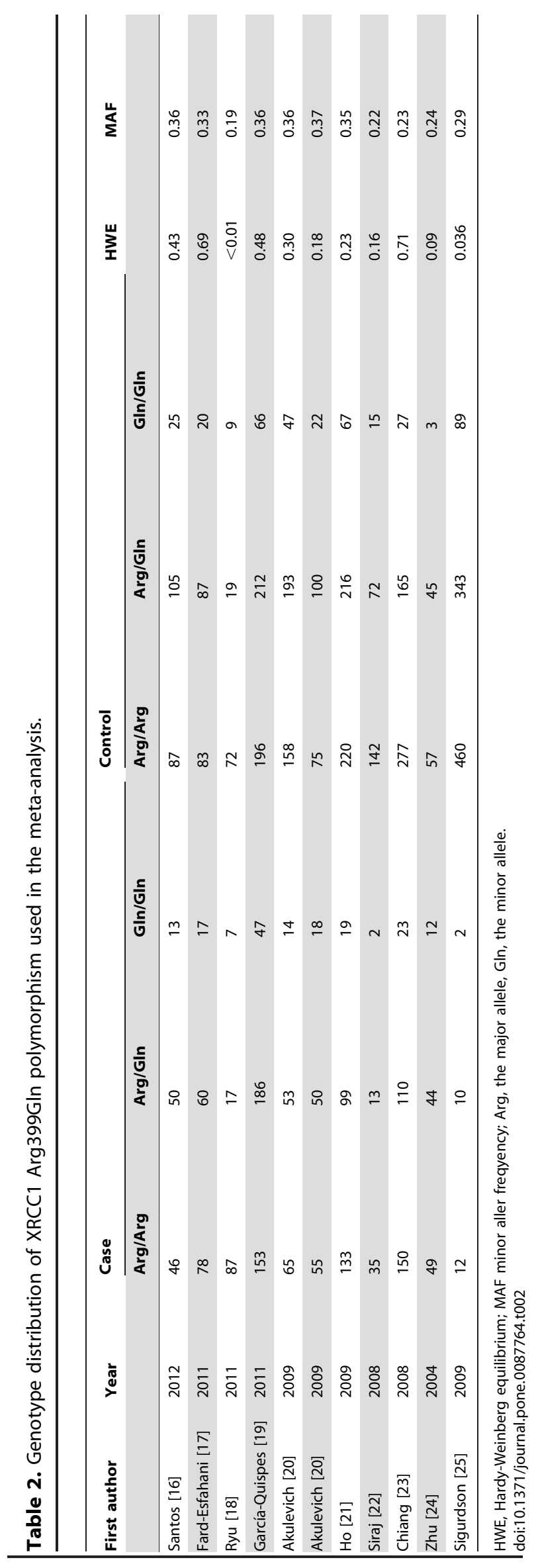

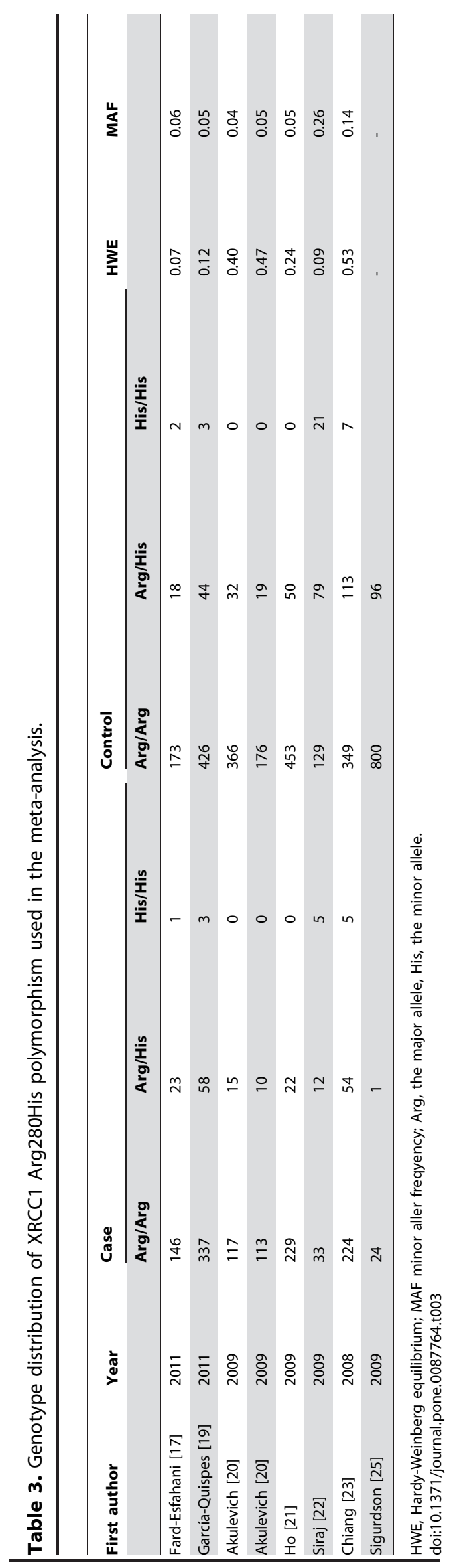




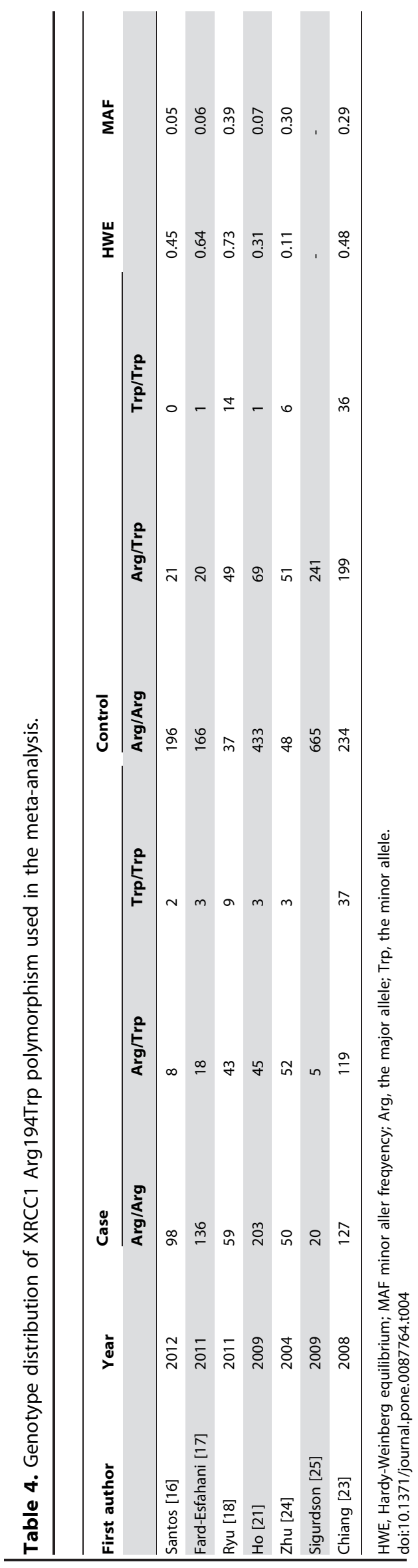

homozygote model, and $P=0.293$ for heterozygote model), and Arg280His $(P=0.656$ for dominant model, $P=0.236$ for recessive model, $P=0.821$ for additive model, $P=0.588$ for homozygote model, and $P=0.992$ for heterozygote model), respectively.

\section{Discussion}

DNA is continuously damaged by endogenous and exogenous mutagens and carcinogens. The damages are fixed by multiple DNA repair pathways including base excision repair, nucleotide excision repair, mismatch repair, and double-strand break repair [15]. Cells with unrepaired DNA damage undergo either apoptosis or unregulated growth to malignancy. A defect or reduced efficiency in repairing DNA damage therefore plays a pivotal role in the development of cancer. One of the DNA repair genes exhibiting polymorphic variation is XRCG1, which is located on chromosome 19q13.2 and encodes a $\mathrm{M}_{\mathrm{r}}$ 70,000 protein [10]. XRCG1 (X-ray cross-complementing group 1 protein) is involved in the repair of DNA base damage and single-strand DNA breaks by binding DNA ligase III at its carboxyl and DNA polymerase $\beta$ and poly (ADP-ribose) polymerase at the site of damaged DNA [11]. Deletion of the XRCG1 gene in mice results in an embryonic lethal phenotype [12]. Chinese hamster ovary cell lines with mutations in the XRCG1 have shown a reduced ability to repair single-strand breaks in DNA and concomitant cellular hypersensitivity to ionizing radiation and alkylating agents [13]. These suggest that XRCCl plays an essential role in the removal of endogenous and exogenous DNA damage. Three polymorphisms in coding regions of the XRCG1 gene at codons 194 (Arg to Trp), 280 (Arg to His), and 399 (Arg to Gln) have been recently identified [14]. A number of epidemiological studies have evaluated the association between XRCG1 Arg399Gln, Arg194Trp, and Arg280His polymorphisms and thyroid cancer risk, but the results remain inconclusive. In order to resolve this conflict, a meta-analysis was performed to examine the association between XRCC1 polymorphisms and thyroid cancer risk, by critically reviewing 11 studies on XRCC1 Arg399Gln (a total of 1729 cases and 3774 controls), 7 studies on Arg 194Trp (1040 cases and 2487 controls), and 8 studies on Arg280His (1432 cases and 3356 controls).

Overall, no significant association was found between XRCC1 Arg399Gln, Arg280His, and Arg194Trp when all the eligible studies were pooled into the meta-analysis. And In the further stratified and sensitivity analyses, significant association was still not found in these three genetic polymorphisms. Zhu et al. [24] in 2004, Santos et al. [16], Sigurdson et al. [25], and Ho et al. [21] reported that the XRCC1 Arg194Trp was not associated with the risk of thyroid cancer. Ryu et al. [18] in 2011, Santos et al. [16], Sigurdson et al. [25], García-Quispes et al. [19], Fard-Esfahani et al. [17], Chiang et al. [23] and Akulevich et al. [20] reported that the XRCCl Arg399Gln polymorphism was not associated with the risk of thyroid cancer. Sigurdson et al. [25], Fard-Esfahani et al. [17] Akulevich et al. [20], and Chiang [23] et al. reported that the XRCG1 Arg280His polymorphism was not associated with the risk of thyroid cancer. The results of our meta-analysis supported the negative association between XRCG1 Arg399Gln, Arg194Trp, and Arg280His polymorphisms and thyroid cancer risk. However, a careful matching should be considered in future larger genetic association studies including multiple ethnic groups. In the present meta-analysis, between-studies heterogeneity was observed for XRCC1 Arg399Gln, Arg280His, and Arg194Trp. The results of meta-regression indicated that ethnicity but not source of controls contributed to substantial heterogeneity among the meta-analysis of Arg280His and Arg399Gln. Hence, the same 


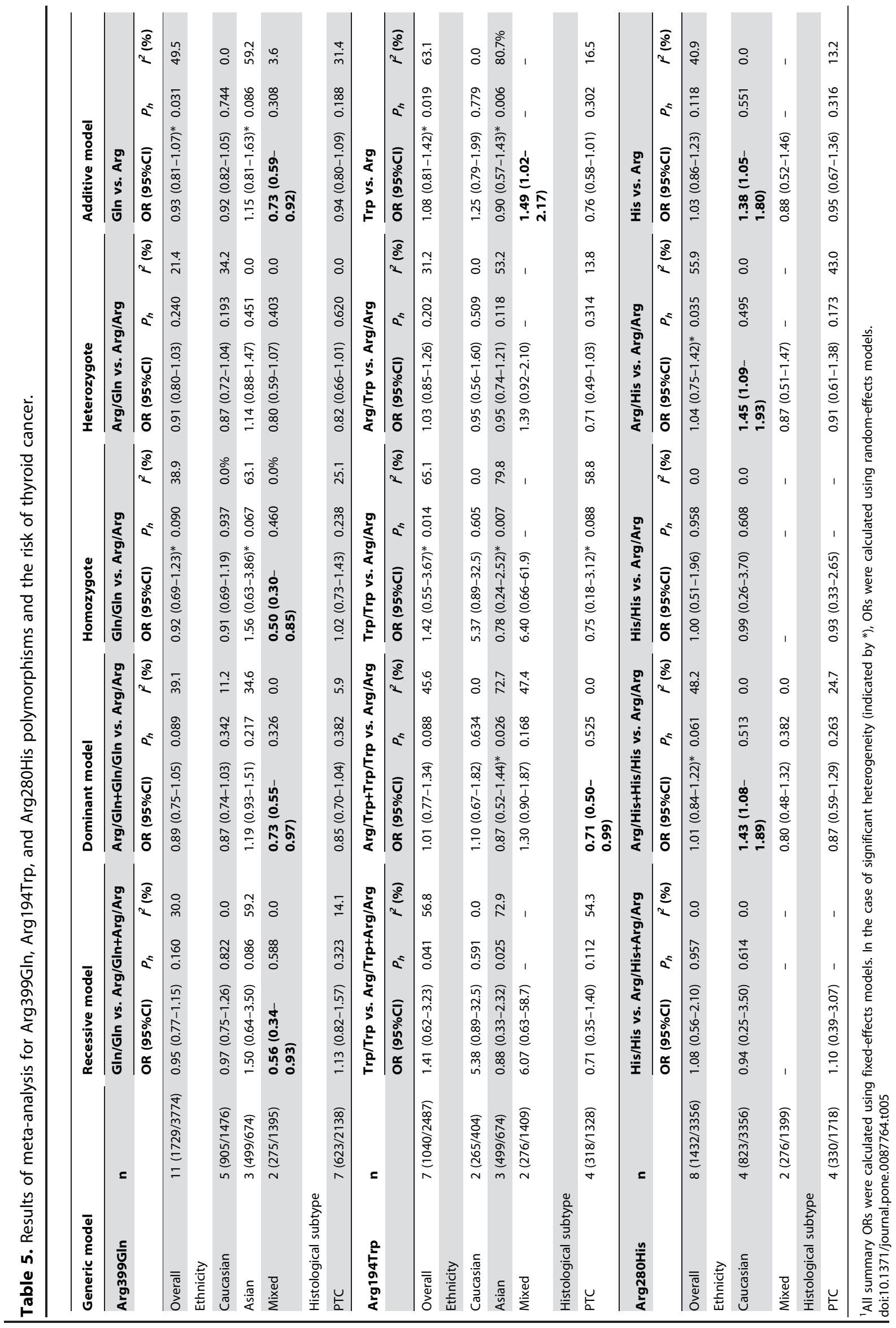



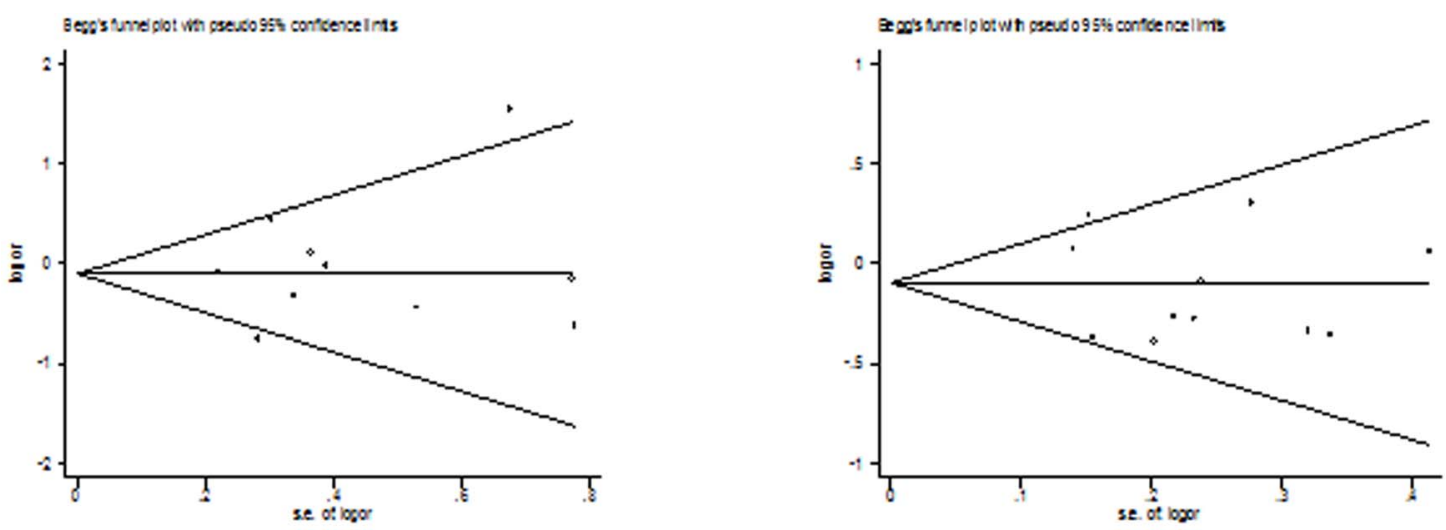

Figure 2. Begg's funnel plot of the meta-analysis of thyroid cancer risk and XRCC1 Arg399Gln polymorphism. (Homozygote model and dominant model).

doi:10.1371/journal.pone.0087764.g002

polymorphisms may play different roles in different ethnicity, because cancer is a complicated multi-genetic disease, and different genetic backgrounds may contribute to the discrepancy. And even more importantly, the low penetrance genetic effects of single polymorphism may largely depend on interaction with other polymorphisms and/or a particular environmental exposure.

Previous meta-analyses on XRCC1 Arg399Gln, Arg194Trp, and Arg280His polymorphisms with thyroid cancer risk showed conflicting results. The study of $\mathrm{Hu}$ et al. [33] suggested that XRCG1 Arg399Gln polymorphism is not associated with differentiated thyroid carcinoma risk, while a decreased risk is observed among Caucasian population. The study of Qian et al. [8] suggested that XRCC1 Arg399Gln polymorphism might be associated with decreased thyroid cancer risk among Caucasians and XRCC1 Arg194Trp may be associated with a tendency for increased thyroid cancer risk in the two larger sample size trials. The study of Bao et al. [9] suggested that Arg280His might contribute to the susceptibility of Differentiated Thyroid Carcinoma (DTC) among Caucasians, whereas it might provide protective effects in Asians against the risk of DTC. Additionally, their results supported the protective role of Arg 194Trp polymorphism in developing PTC, and showed evidence of an association between Arg399Gln polymorphism and decreased risk of DTC in mixed population. The study of Du et al. [34] suggested that XRGC1 Arg194Trp may be a risk factor for DTC development.
The study of Wang et al. [35] demonstrated that the XRCC1 Arg399Gln, Arg194Trp, and Arg280His may be associated with developing of thyroid cancer. However, the results of the present meta-analysis are not in accordance with those reported by the previous meta-analysis [8,9,33-35]. Our meta-analysis indicates that XRCG1 Arg399Gln, Arg280His, and Arg194Trp are not associated with thyroid cancer. Our results seem to confirm and establish the trend in the meta-analysis of the XRCG1 Arg399Gln, Arg280His, and Arg194Trp polymorphisms because this metaanalysis performed a more complete sensitivity analysis than the previous meta-analysis [8,9,33-35]. We found that previous metaanalysis [8,9,33-35] did not seriously perform the sensitivity analysis. hence, their meta-analysis results may be inaccurate.

There are several limitations in this meta-analysis. First, the controls were not uniformly defined. Although most of them were common populations, some controls were population-based; other controls were hospital-based. Hence, non-differential misclassification bias is possible. Second, in the subgroup analysis may have had insufficient statistical power to check an association, Third, we were also unable to examine the interactions among geneenvironment, lacking of the original data of the included studies limited our further evaluation of potential interactions, which may be an important component of the association between XRCG1 Arg399Gln, Arg280His, and Arg194Trp polymorphisms and environment and thyroid cancer risk. Four, it was much difficult
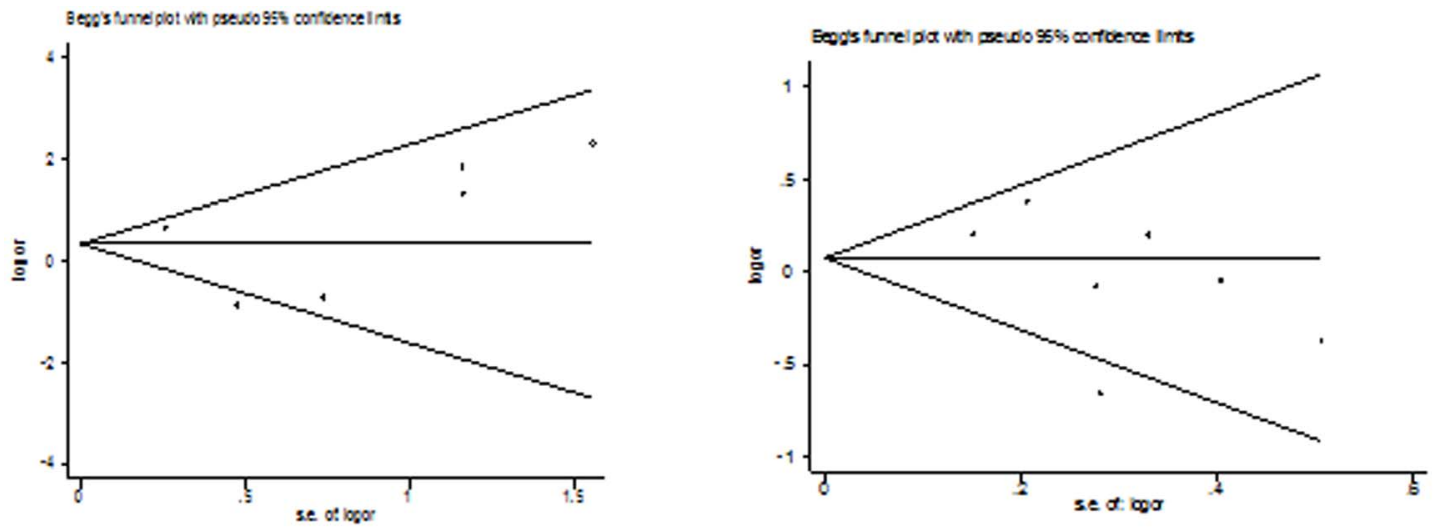

Figure 3. Begg's funnel plot of the meta-analysis of thyroid cancer risk and XRCC1 Arg194Trp polymorphism. (Homozygote model and dominant model).

doi:10.1371/journal.pone.0087764.g003 

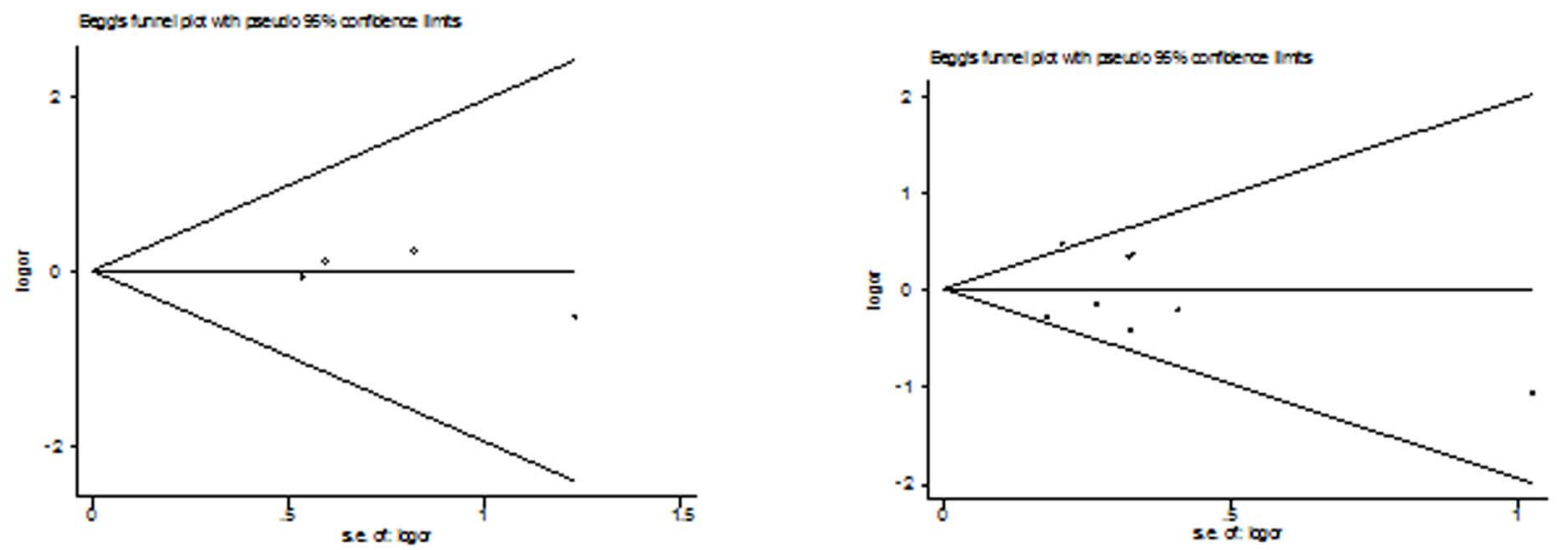

Figure 4. Begg's funnel plot of the meta-analysis of thyroid cancer risk and XRCC1 Arg194Trp polymorphism. (Homozygote model and dominant model). doi:10.1371/journal.pone.0087764.g004

to get the all articles published in various language. Last, our results were based on unadjusted published estimates. Because of data limitations, we were unable to adjust them such as age and alcohol consumption et al. Our meta-analysis also has several strengths. First, a meta-analysis of the association of XRCG1 Arg399Gln, Arg280His, and Arg194Trp polymorphisms with thyroid cancer risk is statistically more powerful than any single study. Second, the quality of eligible studies included in current meta-analysis was satisfactory and met our inclusion criterion.

In summary, this meta-analysis indicates that XRCC1 Arg399Gln, Arg280His, and Arg194Trp are not associated with thyroid cancer. However, it is necessary to conduct large sample studies using standardized unbiased genotyping methods, homogeneous cancer patients and well-matched controls. Moreover, further studies estimating the effect of gene-gene and gene-

\section{References}

1. Wein RO, Weber RS (2005) Contemporary management of differentiated thyroid carcinoma. Otolaryngol Clin North Am 38:161-178.

2. Preston-Martin S, Franceschi S, Ron E, Negri E (2003) Thyroid cancer pooled analysis from 14 case-control studies: what have we learned? Cancer Causes Control 14:787-789.

3. Akulevich NM, Saenko VA, Rogounovitch TI, Drozd VM, Lushnikov EF, et al. (2009) Polymorphisms of DNA damage response genes in radiation-related and sporadic papillary thyroid carcinoma. Endocr Relat Cancer 16:491-503.

4. Thacker J, Zdzienicka MZ (2003) The mammalian XRCC genes: their roles in DNA repair and genetic stability. DNA Repair (Amst) 2:655-672.

5. Caldecott KW, Aoufouchi S, Johnson P, Shall S (1996) XRCC1 polypeptide interacts with DNA polymerase beta and possibly poly (ADP-ribose) polymerase, and DNA ligase III is a novel molecular nick-sensor in vitro. Nucleic Acids Res 24:4387-4397.

6. Caldecott KW (2003) XRCC1 and DNA strand break repair. DNA Repair (Amst) 2:955-969.

7. Shen MR, Jone IM, Mohrenweiser H (1998) Nonconservative amino acid substitution variants exist at polymorphic frequency in DNA repair genes in healthy humans. Cancer Res 58:604-608.

8. Qian K, Liu KJ, Xu F, Chen XY, Chen GN, et al. (2012) X-Ray Repair CrossComplementing Group 1(XRCC1) Genetic Polymorphisms and Thyroid Carcinoma Risk: a Meta-Analysis. Asian Pac J Cancer Prev 13:6385-6390.

9. Bao Y, Jiang L, Zhou JY, Zou JJ, Zheng JY, et al. (2013) XRCCl Gene Polymorphisms and the Risk of Differentiated Thyroid Carcinoma (DTC): A Meta-Analysis of Case-Control Studies. PLoS One 2013;8:e64851.

10. Lamerdin J, Montgomery M, Stilwagen S, Scheidecker L, Tebbs R, et al. (1995) Genomic sequence comparison of the human and mouse XRCC1 DNA repair gene regions. Genomics 25:547-554.

11. Caldecott KW, Aoufouchi S, Johnson P, Shall S (1996) XRCG1 polypeptide interacts with DNA polymerase? and possibly poly(ADP-ribose) polymerase, and DNA ligase III is a novel molecular "nick sensor" in vitro. Nucleic Acids Res 24:4387-4397.

12. Tebbs RS, Flannery ML, Meneses JJ, Hartmann A, Tucker JD, et al. (1999) Requirement for the Xrccl DNA base excision repair gene during early mouse development. Dev Biol 208:513-529. environment interactions may eventually lead to our better, comprehensive understanding of the association between the XRCG1 Arg399Gln, Arg280His, and Arg194Trp polymorphisms and thyroid cancer risk.

\section{Supporting Information}

\section{Checklist S1 PRISMA Ghecklist.}

(DOC)

\section{Author Contributions}

Conceived and designed the experiments: FFW HWS GJQ. Performed the experiments: FFW HWS. Analyzed the data: XFH FFW. Contributed reagents/materials/analysis tools: FFW XFH GJQ. Wrote the paper: FFW $\mathrm{XFH}$.

13. Shen MR, Zdzienicka MZ, Mohrenweiser H, Thompson LH, Thelen MP (1998) Mutations in hamster single-strand break repair gene XRCC1 causing defective DNA. Nucleic Acids Res 26:1032-1037.

14. Shen MR, Jones IM, Mohrenweiser H (1998) Nonconservative amino acid substitution variants exist at polymorphic frequency in DNA repair genes in healthy humans. Cancer Res 58:604-608.

15. Goode EL, Ulrich CM, Potter JD (2002) Polymorphisms in DNA repair genes and associations with cancer risk. Cancer Epidemiol Biomark Prev 11:15131530 .

16. Santos LS, Branco SC, Silva SN, Azevedo AP, Gil OM, et al. (2012) Polymorphisms in base excision repair genes and thyroid cancer risk. Oncol Rep 28:1859-1868.

17. Fard-Esfahani P, Fard-Esfahani A, Fayaz S, Ghanbarzadeh B, Saidi P, et al. (2011) Association of Arg194Trp, Arg280His and Arg399Gln polymorphisms in $\mathrm{X}$-ray repair cross-complementing group 1 gene and risk of differentiated thyroid carcinoma in Iran. Iran Biomed J 15:73-78.

18. Ryu RA, Tae K, Min HJ, Jeong JH, Cho SH, et al. (2011) XRGC1 polymorphisms and risk of papillary thyroid carcinoma in a Korean sample. J Korean Med Sci 26:991-995.

19. García-Quispes WA, Pérez-Machado G, Akdi A, Pastor S, Galofré P, et al. (2011) Association studies of OGG1, XRCG1, XRGC2 and XRGC3 polymorphisms with differentiated thyroid cancer. Mutat Res 709-710:67-72.

20. Akulevich NM, Saenko VA, Rogounovitch TI, Drozd VM, Lushnikov EF, et al. (2009) Polymorphisms of DNA damage response genes in radiation-related and sporadic papillary thyroid carcinoma. Endocr Relat Cancer 16:491-503.

21. Ho T, Li G, Lu J, Zhao G, Wei Q et al. (2009) Association of XRCG1 polymorphisms and risk of differentiated thyroid carcinoma: a case-control analysis. Thyroid 19:129-135.

22. Siraj AK, Al-Rasheed M, Ibrahim M, Siddiqui K, Al-Dayel F, et al. (2008) RAD52 polymorphisms contribute to the development of papillary thyroid cancer susceptibility in Middle Eastern population. J Endocrinol Invest 31:893899.

23. Chiang FY, Wu CW, Hsiao PJ, Kuo WR, Lee KW, et al. (2008) Association between polymorphisms in DNA base excision repair genes XRCC1, APE1, and ADPRT and differentiated thyroid carcinoma. Clin Cancer Res 14:5919-5924. 
24. Zhu QX, Bian JC, Shen Q Jiang F, Tang HW, et al. (2004) Genetic polymorphisms in X-ray repair cross-complementing gene 1 and susceptibility to papillary thyroid carcinoma. Zhonghua Liu Xing Bing Xue Za Zhi 25:702-705.

25. Sigurdson AJ, Land CE, Bhatti P, Pineda M, Brenner A, et al. (2009) Thyroid nodules, polymorphic variants in DNA repair and RET-related genes, and interaction with ionizing radiation exposure from nuclear tests in Kazakhstan. Radiat Res 171:77-88.

26. Davey SG, Egger M (1997) Meta-analyses of randomized controlled trials. Lancet 350:1182.

27. Higgins JP, Thompson SG, Deeks JJ, Altman DG (2003) Measuring inconsistency in meta-analysis. Br Med J 327:557-560.

28. Mantel N, Haenszel W (1959) Statistical aspects of the analysis of data from retrospective studies of disease. Natl Cancer Inst 22:719-748.

29. DerSimonian R, Laird N (1986) Meta-analysis in clinical trials. Control Clin Trials 7:177-188.
30. Begg CB, Mazumdar M (1994) Operating characteristics of a rank correlation test for publication bias. Biometrics 50:1088-1101.

31. Egger M, Smith DG, Schneider M, Minder C (1997) Bias in meta-analysis detected by a simple, graphical test. Br Med J 315:629-634.

32. Neta G, Brenner AV, Sturgis EM, Pfeiffer RM, Hutchinson AA, et al. (2011) Common genetic variants related to genomic integrity and risk of papillary thyroid cancer. Carcinogenesis 32: 1231-1237.

33. Hu Z, Hu X, Long J, Su L, Wei B (2013) XRCC1 polymorphisms and differentiated thyroid carcinoma risk: a meta-analysis. Gene 528:67-73.

34. Du Y, Han LY, Li DD, Liu H, Gao YH, et al. (2013) Associations between XRCC1 Arg399Gln, Arg194Trp, and Arg280His polymorphisms and risk of differentiated thyroid carcinoma: a meta-analysis. Asian Pac J Cancer Prev 14:5483-5487.

35. Wang C, Ai Z (2014) Association of XRCC1 polymorphisms with thyroid cancer risk. Tumour Biol 35:4791-4797. 